\title{
Balancing Between Cognitive and Semantic Acceptability of Arguments
}

\author{
Hiroyuki Kido $^{1}$ and Keishi Okamoto ${ }^{2}$ \\ 1 Institute of Logic and Cognition, Sun Yat-sen University \\ kido@mail.sysu.edu.cn \\ 2 Department of Information Systems, National Institute of Technology, Sendai College \\ okamoto@sendai-nct.ac.jp
}

\begin{abstract}
This paper addresses the problem concerning approximating human cognitions and semantic extensions regarding acceptability status of arguments. We introduce three types of logical equilibriums in terms of satisfiability, entailment and semantic equivalence in order to analyse balance of human cognitions and semantic extensions. The generality of our proposal is shown by the existence conditions of equilibrium solutions. The applicability of our proposal is demonstrated by the fact that it detects a flaw of argumentation actually taking place in an online forum and suggests its possible resolution.
\end{abstract}

\section{Introduction}

Argumentation is a verbal, social and rational activity [17]. It is also a daily activity in the sense that thinking hard has almost the same meaning as arguing oneself carefully. Meanwhile, it is unclear how one should engage in and facilitate rational argumentation. This is because there seems to be no universal evaluation standard telling what rational argumentation is. However, in this paper, we argue that one aspect of such standards is given by acceptability semantics, e.g., Dung's acceptability semantics [13], as well as more advanced ones, e.g., stage semantics [18], semi-stable semantics [10], ideal semantics [14], CF2 semantics [5] and prudent semantics [12].

These and most acceptability semantics have the language independence principle [4] meaning that they intrinsically refer not to contents, e.g., sentences and words, existing in arguments but to relations, e.g., attack and support relations, existing between arguments when they define acceptability status of arguments. We thus call them (formal) semantic acceptability in the sense that such contents do not directly affect evaluation. ${ }^{3}$ By contrast, we call human judgment based on contents of arguments cognitive acceptability. Both acceptability might be the same in some situations, but different and moreover incompatible in other situations. As an example, let us consider the following two conflicting arguments $A$ and $B$ attached with their votes.

Argument A (having 1001 positive votes) Veterinarians should have a right to apply animal euthanasia to pets because they must respect pet owners' will.

\footnotetext{
${ }^{3}$ We recognise that sentences existing in arguments indirectly affect semantic acceptability in the sense that they define relations among arguments.
} 
Argument B (having 1002 positive votes) It is not acceptable to allow veterinarians to kill innocent pets because they cannot confirm pets' own will.

In terms of semantic acceptability, e.g., Dung's semantics, $A$ and $B$ cannot be acceptable at the same time because they are in conflict, i.e., attack each other. However, in terms of cognitive acceptability, $A$ and $B$ should be acceptable at the same time because they have a lot of positive votes. Now, does this situation show that agents who vote for both $A$ and $B$ are irrational or the truth is that there is no attack relation between these arguments? We think neither is true. The conflict is a result of rational judgment caused by focusing on different sources of information: on the one hand, a relation between arguments, and on the other hand, contents of arguments. Then, would it be reasonable to leave the conflict as it is, as the existing contradiction? We think that the acceptability should be at least compatible each other. Therefore, in this paper we investigate what kind of difference exists between cognitive and semantic acceptability, and how we should approximate and resolve them.

A difficulty associated with these questions is how to make a detailed analysis of the difference in a unified way. Our novel approach is to introduce equilibriums to analyse balances between cognitive and semantic acceptability using an entailment relation of propositional logic. In this paper, we divide the difference into three types: satisfiability, entailment and semantic equivalence. We formalise a detection of unsatisfiability, and resolutions of satisfiability, entailment and semantic equivalence. We show that there always exist contraction-based satisfiability, entailment and semantic-equivalence resolutions, and there always exist their expansion-based resolutions under the condition that agent's perceptions do not violate conflict-freeness of acceptability semantics.

The contributions of this paper are as follows. Firstly, to the best of our knowledge, this is the first paper arguing for the need for balancing between agent's perceptions and semantic extensions. Although some recent work covers a change of agent's perceptions and semantic extension, it handles a change of either of them and their balanced change is outside its scope (See Section 5). By benefitting from expression and analytical power of propositional logic, this paper makes it possible to handle sensitively balanced contractions and expansions of them (See Section 3). Secondly, this paper relates to both theory and practice of computational argumentation. In fact, we demonstrate how our theoretical concepts, i.e., equilibrium notions, highlight flaws of argument actually taking place in an online forum, and suggest their possible resolutions (See Section 4).

\section{Preliminaries}

Dung's acceptability semantics [13] is a general and abstract theory of formal argumentation. It is general in the sense that the semantics reinterprets consequence relations of various approaches for nonmonotonic reasoning. It is abstract in the sense that the semantics is defined on a directed graph, called an abstract argumentation framework, denoted by $A F$. AF is defined as a pair $\langle A r g s, A t t s\rangle$ where Args is a set of arguments and Atts $\subseteq$ Args $\times$ Args is a binary relation on Args $((A, B) \in$ Atts means " $A$ attacks $B$ "). Suppose $A \in A$ Args and $S \subseteq$ Args. $S$ attacks $A$ iff some member of $S$ attacks $A$. $S$ is conflict-free iff $S$ attacks none of its members. $S$ defends $A$ iff $S$ is conflict-free and $S$ attacks all arguments attacking $A$. Given $A F$, 
Dung's acceptability semantics defines four kinds of sets, called extensions, of acceptable arguments. $S$ is a complete extension iff $S$ is a fixed point of the function $F:$ Pow $($ Args $) \rightarrow$ Pow (Args) where $F(S)=\{a \mid S$ defends $a\}$ and Pow(Args) is the power set of Args. $S$ is a grounded (resp. preferred) extension iff it is the minimum (resp. a maximal) complete extension with respect to set inclusion. $S$ is a stable extension iff it is a complete extension attacking all members in Args $\backslash S$. We assume functions $\arg (A F)$ and $\operatorname{att}(A F)$ to refer to the set of arguments Args and attack relations Atts of $A F$, respectively.

Example 1. Let $A F$ be the argumentation framework where $\arg (A F)=\{A, B, C$, $D\}$, and $\operatorname{att}(A F)=\{(B, C),(C, B),(C, D),(D, D)\}$. The graph representation of $A F$ and four types of extensions are described in the left and right below, respectively.

$$
\begin{aligned}
& \text { - Preferred extensions: }\{A, B\},\{A, C\} \\
& A F: A \quad B \leftrightarrow C \rightarrow D^{2} \quad \begin{array}{ll}
\text { - Stable extension: }\{A, C\} \\
\text { - Grounded extension: }\{A\}
\end{array} \\
& \text { - Complete extensions: }\{A\},\{A, B\},\{A, C\}
\end{aligned}
$$

\section{Equilibrium-Based Resolutions}

\subsection{Semantic and cognitive acceptabilities}

We introduce a propositional language associated with an abstract argumentation framework. It is used to describe acceptability status of arguments in the framework.

Definition 1 (Language). Let $A F$ be an abstract argumentation framework. A propositional language $L_{A F}$ associated with $A F$ is defined as follows. For all arguments $X \in \arg (A F), x$ is an atomic formula of $L_{A F}$. When $x$ and $y$ are formulas of $L_{A F}$, $(x \wedge y),(x \vee y),(x \rightarrow y)$ and $\neg x$ are formulas of $L_{A F}$.

For all atomic formulas $x \in L_{A F}$, " $x$ is true" intuitively means "argument $X$ is acceptable." Given an extension, arguments are acceptable if and only if they are members of the extension. Different two extensions define alternative memberships. Therefore, a logical expression of extensions is defined as follows.

Definition 2 (Extensions). Let $A F$ be an abstract argumentation framework and $\mathcal{E}$ be a set of extensions of $A F$. A logical expression of $\mathcal{E}$ is given as follows. ${ }^{4}$

$$
\left\{\bigvee_{E \in \mathcal{E}}\left(\bigwedge_{X \in E} x \wedge \bigwedge_{X \in A r g s \backslash E} \neg x\right)\right\}
$$

Example 1 (continued). Logical expressions of all types of extensions of $A F$ are given as follows.

- Preferred extensions : $\{(a \wedge b \wedge \neg c \wedge \neg d) \vee(a \wedge \neg b \wedge c \wedge \neg d)\}$

- Stable extension: $\{a \wedge \neg b \wedge c \wedge \neg d\}$

- Grounded extension: $\{a \wedge \neg b \wedge \neg c \wedge \neg d\}$

\footnotetext{
${ }^{4}$ The exclusive OR is strictly appropriate. However, we use OR because of their equivalence.
} 
- Complete extensions: $\{(a \wedge \neg b \wedge \neg c \wedge \neg d) \vee(a \wedge b \wedge \neg c \wedge \neg d) \vee(a \wedge \neg b \wedge c \wedge \neg d)\}$

In what follows, we assume a fixed and arbitrary abstract argumentation framework $A F$ and acceptability semantics $\varepsilon$. We use function $\varepsilon(A F)$ to refer to the logical expression of the set of extensions of $A F$ with respect to $\varepsilon$. We assume a fixed and arbitrary consistent set $\Sigma \subseteq L_{A F}$ of formulas. On the one hand, $\varepsilon(A F)$ is used to represent semantic extensions, and on the other hand, $\Sigma$ is used to represent cognitive extensions.

\subsection{Satisfiability resolution}

This subsection discusses how to detect and resolve incompatibility between cognitive acceptability $\Sigma$ and semantic acceptability $\varepsilon(A F)$. A minimally unsatisfiable set is a minimal subset of cognitive acceptability that causes incompatibility with semantic acceptability.

Definition 3 (Minimally unsatisfiable set). $\Sigma_{\downarrow} \subseteq L_{A F}$ is a minimally unsatisfiable set with respect to $A F$ iff $\Sigma_{\downarrow}$ is a minimal subset of $\Sigma$ with respect to $\supseteq$ such that $\varepsilon(A F) \cup \Sigma_{\downarrow}$ is unsatisfiable.

An order relation between abstract argumentation frameworks is introduced to refer to maximality and minimality of frameworks.

Definition 4 (Subframework/superframework). Let $A F_{i}$ and $A F_{j}$ be abstract argumentation frameworks. $A F_{i}$ is a subframework of $A F_{j}$ (or $A F_{j}$ is a superframework of $\left.A F_{i}\right)$, denoted by $A F_{i} \sqsubseteq A F_{j}$, iff $\arg \left(A F_{i}\right) \subseteq \arg \left(A F_{j}\right)$ and $\operatorname{att}\left(A F_{i}\right) \subseteq \operatorname{att}\left(A F_{j}\right)$.

An unsatisfiable core is intuitively a minimal subframework preserving incompatibility existing between $A F$ and $\Sigma$.

Definition 5 (Unsatisfiable core). An abstract argumentation framework $A F_{\downarrow}$ is an unsatisfiable core of $A F$ with respect to $\Sigma$ iff there is a minimally unsatisfiable set $\Sigma_{\downarrow}$ with respect to $A F$, and $A F_{\downarrow}$ is a minimal subframework of $A F$ such that $\Sigma_{\downarrow}$ is a minimally unsatisfiable set with respect to $A F_{\downarrow}$.

Example 2. Let us consider the following argumentation framework $A F$ and the satisfiable set $\Sigma=\{a, a \rightarrow b, \neg c\} \subseteq L_{A F}$.

$$
A F: A \longleftrightarrow B \longleftrightarrow C
$$

Complete extensions of $A F$ is $\{(a \wedge \neg b \wedge c) \vee(\neg a \wedge b \wedge \neg c) \vee(\neg a \wedge \neg b \wedge \neg c)\}$. In this situation, $\Sigma_{1}=\{a, a \rightarrow b\}$ and $\Sigma_{2}=\{a, \neg c\}$ are minimally unsatisfiable sets with respect to $A F$. The following $A F_{1}$ and $A F_{2}$ are the unsatisfiable cores of $A F$ with respect to $\Sigma$.

$$
A F_{1}: A \longleftrightarrow B
$$

A question here is how to resolve incompatibility between cognitive and semantic acceptability. When we consider the situation where semantic acceptability imposes cognitive acceptability to change, a possible resolution is to change the cognitive acceptability. A maximally satisfiable set is a maximal subset of the cognitive acceptability that causes no incompatibility with the semantic acceptability. 
Definition 6 (Maximally satisfiable set). $\Sigma_{\downarrow} \subseteq \Sigma$ is a maximally satisfiable set with respect to $A F$ iff $\Sigma_{\downarrow}$ is a maximal subset of $\Sigma$ with respect to $\supseteq$ such that $\varepsilon(A F) \cup \Sigma_{\downarrow}$ is satisfiable.

Meanwhile, when we consider the situation where cognitive acceptability imposes on semantic acceptability to change, a possible resolution is to contract or expand AFs. Each maximally and minimally satisfiable framework represents a contraction and expansion of AFs, respectively.

Definition 7 (Maximally/minimally satisfiable framework). Let $A F_{\uparrow}$ be an abstract argumentation framework. $A F_{\uparrow}$ is a maximally (resp. minimally) satisfiable framework with respect to $\Sigma$ iff $A F_{\uparrow}$ is a maximal subframework (resp. minimal superframework) of $A F$ with respect to $\sqsupseteq$ such that $\varepsilon\left(A F_{\uparrow}\right) \cup \Sigma$ is satisfiable.

A contraction is appropriate when an AF is uncertain in the sense that existence of arguments and attacks is unclear. An expansion is appropriate when an AF is incomplete in the sense that there can exist additional arguments or attacks. A satisfiability resolution is defined as an equilibrium point between cognitive and semantic acceptability.

Definition 8 (Satisfiability resolutions). Let $A F_{\uparrow}$ be an abstract argumentation framework and $\Sigma_{\downarrow} \subseteq \Sigma$ be a set. The pair $\left(A F_{\uparrow}, \Sigma_{\downarrow}\right)$ is a satisfiability resolution of $(A F, \Sigma)$ iff $\Sigma_{\downarrow}$ is a maximally satisfiable set with respect to $A F_{\uparrow}$ and $A F_{\uparrow}$ is a maximally or minimally satisfiable framework with respect to $\Sigma_{\downarrow}$.

In particular, we call satisfiability resolutions contraction-based when they are based on maximally satisfiable frameworks, and expansions-based when they are based on minimally satisfiable frameworks.

Example 2 (continued). All possible contraction-based and expansion-based satisfiability resolutions of $(A F, \Sigma)$ are described in the left and right below, respectively.

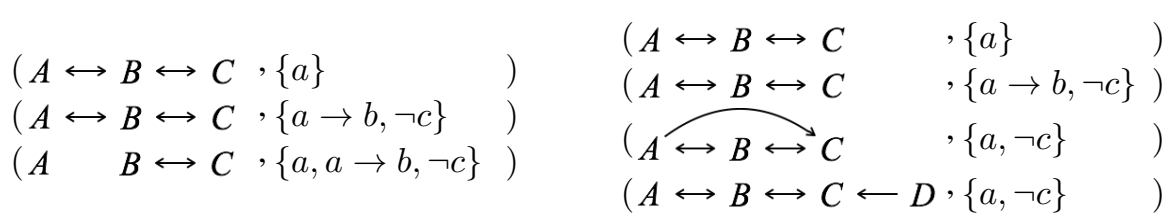

Here, the domain of discourse for possible expansions of $A F$ is assumed to be given by the complete graph $A F_{D}$ as follows.

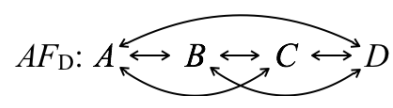

\subsection{Entailment resolution}

This subsection deals with the issue that semantic acceptability cannot entail cognitive acceptability. The first approach for solving this problem is to change cognitive acceptability. We define a maximally entailed set as follows. 
Definition 9 (Maximally entailed set). $\Sigma_{\downarrow} \subseteq \Sigma$ is a maximally entailed set with respect to $A F$ iff $\Sigma_{\downarrow}$ is a maximal subset of $\Sigma$ with respect to $\supseteq$ such that $\varepsilon(A F) \models \Sigma_{\downarrow}$ holds. ${ }^{5}$

The second approach is to contract or expand argumentation frameworks defining semantic acceptability. Both maximally and minimally entailing frameworks are minimal changes of AFs whose semantic acceptability entails cognitive acceptability.

Definition 10 (Maximally/minimally entailing framework). Let $A F_{\uparrow}$ be an abstract argumentation framework. $A F_{\uparrow}$ is a maximally (resp. minimally) entailing framework with respect to $\Sigma$ iff $A F_{\uparrow}$ is a maximal subframework (resp. minimal superframework) of $A F$ with respect to $\sqsupseteq$ such that $\varepsilon\left(A F_{\uparrow}\right) \models \Sigma$ holds.

An entailment resolution is defined as an equilibrium point between the acceptabilities.

Definition 11 (Entailment resolutions). Let $A F_{\uparrow}$ be an abstract argumentation framework and $\Sigma_{\downarrow} \subseteq \Sigma$ be a set. The pair $\left(A F_{\uparrow}, \Sigma_{\downarrow}\right)$ is an entailment resolution of $(A F, \Sigma)$ iff $\Sigma_{\downarrow}$ is a maximally entailed set with respect to $A F_{\uparrow}$ and $A F_{\uparrow}$ is a maximally or minimally entailing framework with respect to $\Sigma_{\downarrow}$.

We call entailment resolutions contraction-based (resp. expansion-based) when they are based on maximally (resp. minimally) entailing frameworks.

Example 2 (continued). All possible contraction-based and expansion-based entailment resolutions of $(A F, \Sigma)$ are described in the left and right below, respectively.

$$
\begin{aligned}
& (A \leftrightarrow B \longleftrightarrow C, \emptyset \\
& (A \longrightarrow B \longleftrightarrow C,\{a\} \\
& (A \longleftrightarrow B \longleftarrow C,\{a\} \\
& (A \longleftarrow B \longrightarrow C,\{a \rightarrow b, \neg c\} \\
& (A \quad B \rightarrow C,\{a, a \rightarrow b, \neg c\})
\end{aligned}
$$

$$
\begin{aligned}
& (A \leftrightarrow B \leftrightarrow C \quad, \emptyset \quad) \\
& (A \leftrightarrow B \leftrightarrow C \longleftarrow D \quad,\{\neg c\} \quad) \\
& (A \leftrightarrow B \leftrightarrow C D D,\{a \rightarrow b\} \quad) \\
& (A \hookleftarrow B \hookleftarrow C \longleftarrow D \quad,\{a \rightarrow b, \neg c\}) \\
& (A \leftrightarrow B \leftrightarrow C \quad D,\{a\} \quad) \\
& (A \longleftrightarrow B \longleftarrow C \longleftarrow D \quad,\{a, \neg c\} \quad)
\end{aligned}
$$

\subsection{Semantic equivalence resolution}

This subsection asks how to make cognitive and semantic acceptability coincide with each other. The first approach is to change cognitive acceptability. A maximally (resp. minimally) equivalent set is a maximal subset (resp. minimal superset) of the cognitive acceptability that is equivalent to the semantic acceptability.

Definition 12 (Maximally/minimally equivalent set). $\Sigma_{\uparrow} \subseteq L_{A F}$ is a maximally (resp. minimally) equivalent set with respect to $A F$ iff $\Sigma_{\uparrow}$ is a maximal subset (resp. minimal superset) of $\Sigma$ with respect to $\supseteq$ such that $\Sigma_{\uparrow} \Leftrightarrow \varepsilon(A F)$ holds. $^{6}$

\footnotetext{
${ }^{5} \varepsilon(A F) \models \Sigma_{\downarrow}$ denotes that, for all $x \in L_{A F}$, if $x \in \Sigma_{\downarrow}$ then $\varepsilon(A F) \models x$.

${ }^{6} \Sigma_{\downarrow} \Leftrightarrow \varepsilon(A F)$ denotes that $\Sigma_{\downarrow} \mid=\varepsilon(A F)$ and $\varepsilon(A F) \mid=\Sigma_{\downarrow}$ hold.
} 
The second approach is to change AFs defining semantic acceptability. Both maximally and minimally equivalent frameworks are minimal changes of $A F$ whose semantic acceptability is equivalent to the cognitive acceptability.

Definition 13 (Maximally/minimally equivalent framework). Let $A F_{\uparrow}$ be an $a b$ stract argumentation framework. $A F_{\uparrow}$ is a maximally (resp. minimally) equivalent framework with respect to $\Sigma$ iff $A F_{\uparrow}$ is a maximal subframework (resp. minimal superframework) of $A F$ with respect to $\sqsupseteq$ such that $\Sigma \Leftrightarrow \varepsilon\left(A F_{\uparrow}\right)$ holds.

A semantic equivalence resolution is formally defined as an equilibrium point between cognitive and semantic acceptability.

Definition 14 (Semantic equivalence resolutions). Let $A F_{\uparrow}$ be an abstract argumentation framework and $\Sigma_{\uparrow} \subseteq L_{A F}$ be a set. The pair $\left(A F_{\uparrow}, \Sigma_{\uparrow}\right)$ is a semantic equivalence resolution of $(A F, \Sigma)$ iff $\Sigma_{\uparrow}$ is a maximally or minimally equivalent set with respect to $A F_{\uparrow}$ and $A F_{\uparrow}$ is a maximally or minimally equivalent framework with respect to $\Sigma_{\uparrow}$.

Example 2 (continued). The followings are all contraction-based semantic equivalence resolutions of $(A F, \Sigma)$.

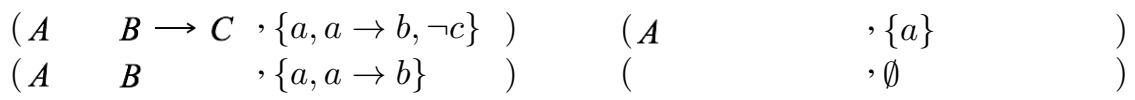

Note that, for example, $(\langle\{A, B, C\},\{(B, C)\}\rangle,\{a, a \rightarrow b, \neg c, b\})$ is not a contractionbased semantic equivalence resolution. Meanwhile, there is no expansion-based semantic equivalence resolution of $(A F, \Sigma)$. So, we here consider another example with $\Sigma^{*}=\{a \vee b, c\}$. We assume a set $\Sigma_{D} \subseteq L_{A F_{D}}$ of a domain discourse for possible expansions of $\Sigma^{*}$ where $A F_{D}$ is the complete graph as shown in the previous example. There is the following expansion-based semantic equivalence resolution of $\left(A F, \Sigma^{*}\right)$.

$$
(A \leftrightarrow B \leftrightarrow C \quad D, \quad\{a \vee b, c, \neg b \wedge d\})
$$

\section{Generality and Applicability}

\subsection{Characterising existence of resolutions}

In this subsection, we show the necessary and sufficient conditions of the existence of our equilibrium-based resolutions. We use the model checking techniques [8] to analyse argumentation theoretic properties of equilibriums. According to the literature, a set $S \subseteq \arg (A F)$ of arguments is conflict-free iff the set $\left\{x \in L_{A F} \mid X \in S\right\}$ is a model of the formula $\Phi_{A F}$ defined as follows.

$$
\Phi_{A F}=\bigwedge_{A \in \arg (A F)}\left(a \rightarrow \bigwedge_{B:(B, A) \in \operatorname{att}(A F)} \neg b\right)
$$

We divide expansion-based and contraction-based resolutions into three types: $\Sigma$ fixation, $\Sigma$ expansion and $\Sigma$ contraction. The first theorem supposes the situation where $\Sigma$ is fixed in expansion-based resolutions. 
Table 1. Summary of the existence of resolutions.

\begin{tabular}{|c|c|c|c|c|c|c|}
\hline & \begin{tabular}{|} 
Expan \\
$\Sigma$ fixation \\
(Theorem 1)
\end{tabular} & $\begin{array}{l}\text { sion-based res } \\
\Sigma \text { expansion } \\
\text { (Theorem 2) }\end{array}$ & $\begin{array}{l}\text { olutions } \\
\Sigma \text { contraction } \\
\text { (Theorem } 3 \text { ) }\end{array}$ & $\begin{array}{c}\text { Contrac } \\
\Sigma \text { fixation } \\
\text { (Theorem 4) }\end{array}$ & $\begin{array}{l}\text { ction-based re } \\
\Sigma \text { expansion } \\
\text { (Theorem 5) }\end{array}$ & $\begin{array}{l}\text { solutions } \\
\Sigma \text { contraction } \\
\text { (Theorem 6) }\end{array}$ \\
\hline Satisfiability & $\checkmark$ (cond.) & $\checkmark$ (cond.) & $\checkmark$ & $\checkmark$ & $\checkmark$ & $\checkmark$ \\
\hline Entailment & $\checkmark$ (cond.) & $\checkmark$ (cond.) & $\checkmark$ & & & $\checkmark$ \\
\hline Equivalence & & $\checkmark$ (cond.) & & & & $\checkmark$ \\
\hline
\end{tabular}

Theorem 1. For any $A F$ and consistent set $\Sigma \subseteq L_{A F}$, there exist abstract argumentation frameworks $A F_{\uparrow}$ such that $\left(A F_{\uparrow}, \Sigma\right)$ are expansion-based satisfiability (entailment, respectively) resolutions of $(A F, \Sigma)$ iff $\Sigma$ do not violate the conflict-free property, i.e., $\Sigma \not \models \neg \Phi_{A F}$.

There does not always exist an expansion-based semantic equivalence resolution $\left(A F_{\uparrow}, \Sigma\right)$ even when $\Sigma$ do not violate the conflict-free property. An example case is when $A F=$ $\langle\{A\}, \emptyset\rangle$ and $\Sigma=\emptyset$. The next two theorems suppose the situations where $\Sigma$ can be expanded and contracted in expansion-based resolutions, respectively.

Theorem 2. For any $A F$ and consistent set $\Sigma \subseteq L_{A F}$, there exist abstract argumentation frameworks $A F_{\uparrow}$ and supersets $\Sigma_{\uparrow}$ of $\Sigma$ such that $\left(A F_{\uparrow}, \Sigma_{\uparrow}\right)$ are expansionbased satisfiability (entailment and semantic equivalence, respectively) resolutions of $(A F, \Sigma)$ iff $\Sigma$ do not violate the conflict-free property, i.e., $\Sigma \not \models \neg \Phi_{A F}$.

Theorem 3. For any $A F$ and consistent set $\Sigma \subseteq L_{A F}$, there exist abstract argumentation frameworks $A F_{\uparrow}$ and subsets $\Sigma_{\downarrow}$ of $\Sigma$ such that $\left(A F_{\uparrow}, \Sigma_{\downarrow}\right)$ are expansion-based satisfiability (entailment, respectively) resolutions of $(A F, \Sigma)$.

Note that Theorems 2 and 3 include Theorem 1, i.e., the case of $\Sigma_{\downarrow}=\Sigma$ and $\Sigma_{\uparrow}=\Sigma$, as special cases, respectively. The next theorem regarding contraction-based resolutions supposes the situation where $\Sigma$ is fixed.

Theorem 4. For any $A F$ and consistent set $\Sigma \subseteq L_{A F}$, there exists an abstract argumentation framework $A F_{\downarrow}$ such that $\left(A F_{\downarrow}, \Sigma\right)$ is a contraction-based satisfiability resolution of $(A F, \Sigma)$.

There does not always exist an entailment and semantic equivalence resolution. For example, when $A F=\langle\{A\}, \emptyset\rangle$ and $\Sigma=\{\neg a\}$, there is neither entailment nor semantic equivalence resolution. The next two theorems suppose the situations where $\Sigma$ can be expanded and contracted in contraction-based resolutions, respectively.

Theorem 5. For any $A F$ and consistent set $\Sigma \subseteq L_{A F}$, there exist an abstract argumentation framework $A F_{\downarrow}$ and a superset $\Sigma_{\uparrow}$ of $\Sigma$ such that $\left(A F_{\downarrow}, \Sigma_{\uparrow}\right)$ is a contractionbased satisfiability resolution of $(A F, \Sigma)$.

Theorem 6. For any $A F$ and consistent set $\Sigma \subseteq L_{A F}$, there exist abstract argumentation frameworks $A F_{\downarrow}$ and subsets $\Sigma_{\downarrow}$ of $\Sigma$ such that $\left(A F_{\downarrow}, \Sigma_{\downarrow}\right)$ are contractionbased satisfiability (entailment and semantic equivalence, respectively) resolutions of $(A F, \Sigma)$. 

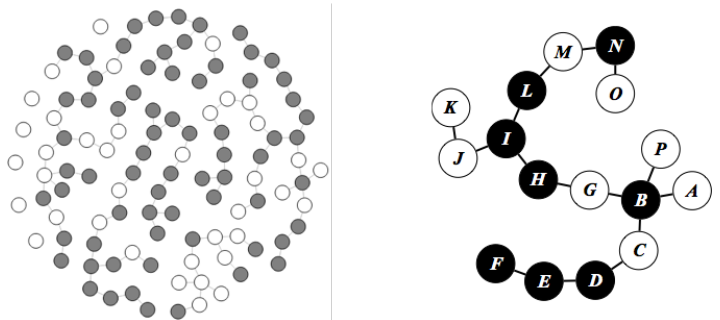

Fig. 1. The left graph shows a whole dispute relations and a summary of positive and negative votes. The right graph shows an argumentation framework constructed from the left graph.

Table 1 shows the summary of the above mentioned theorems in terms of change of $\Sigma$. Symbol " $\checkmark$ " means that there exists a resolution. Meanwhile, "(cond.)" means that there exists a resolution if and only if $\Sigma$ does not violate the conflict-free property. These facts show generality of equilibriums-based approaches in the sense that they have the ability to give resolutions for all types of equilibriums.

\subsection{Application illustration in online forum}

Application potential is one of important criteria for evaluating accuracy of research proposals. This subsection illustrates applicability of our equilibrium based resolutions to argumentation analysis in online debate forums.

CreateDebate [1] is an online forum preserving a number of written arguments, casted votes, and relationships, e.g., dispute, support and clarification relations, among arguments. On the left in Figure 1, we show a whole dispute structure and a summary of users' votes that are both extracted from argument on abortion actually taking place in CreateDebate. The edges represent the whole dispute relations and the white (resp. black) nodes represent that they get user's positive votes above (resp. below) average. On the right in Figure 1, we show a partial graph of the left that especially focuses on discussing a specific subject on abortion. We represent it as an abstract argumentation framework, denoted by $A F$, with a symmetric attack relation, i.e., if $(X, Y) \in \operatorname{att}(A F)$ then $(Y, X) \in \operatorname{att}(A F)$, for all $X, Y \in \arg (A F)$. We assume that $\varepsilon(A F)$ represents the logical expression of the set of preferred extensions. We define agents' cognitions $\Sigma$ from the votes to individual arguments in such a way that $x \in \Sigma$ holds when node $X$ is white and $\neg x \in \Sigma$ holds when node $X$ is black.
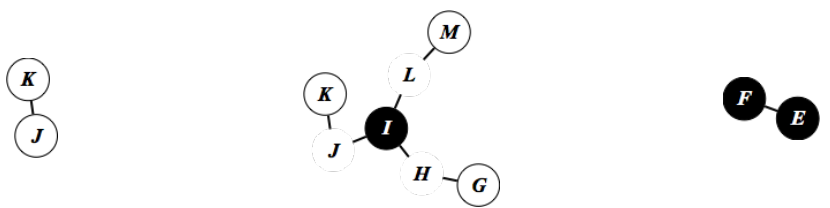

Fig. 2. The inconsistent cores of the argumentation framework shown in the right in Figure 1. 
Figure 2 shows all unsatisfiable cores of $A F$ with respect to $\Sigma .^{7}$ It is observed that $\{k, j\},\{g, \neg i, k, m\}$ and $\{\neg e, \neg f\} \subseteq \Sigma$ are minimally unsatisfiable subsets of $\Sigma$ with respect to $A F$. The inconsistent cores verify consistency between acceptance of arguments coming from the argumentation structure and user's votes. They are useful in the sense that they tell where people should pay attention to resolve incompatibility between agent cognitions and semantic extensions.

A consistent core is obtained by eliminating attacks $(K, J),(J, K),(E, F),(F, E) \in$ $\operatorname{att}(A F)$ from $A F$. Given the $A F$, the left and right graphs in Figure 3 show a contractionbased and an expansion-based satisfiability resolutions, respectively.
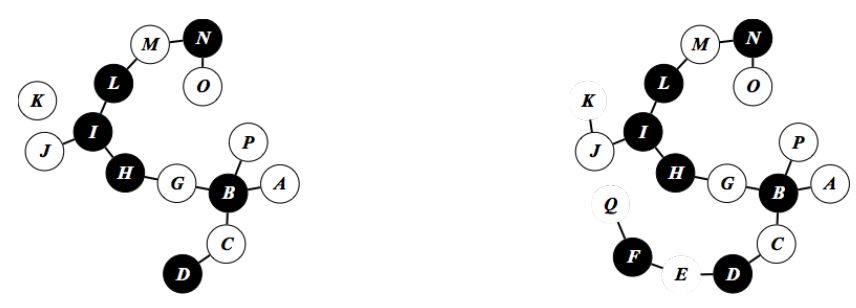

Fig. 3. A contraction-based (left) and an expansion-based (right) satisfiability resolutions.

Note that $\Sigma$ has no change in the contraction-based resolution, although it changes to $\Sigma \backslash\{k, \neg e\}$ in the expansion-based resolution. The satisfiability resolutions facilitate rational argumentation in the sense that they suggest simultaneous updates on both argumentation frameworks and users' cognitions.

Given the expansion-based resolution, Figure 4 shows a contraction-based entailment resolution and a contraction-based semantic equivalence resolution.
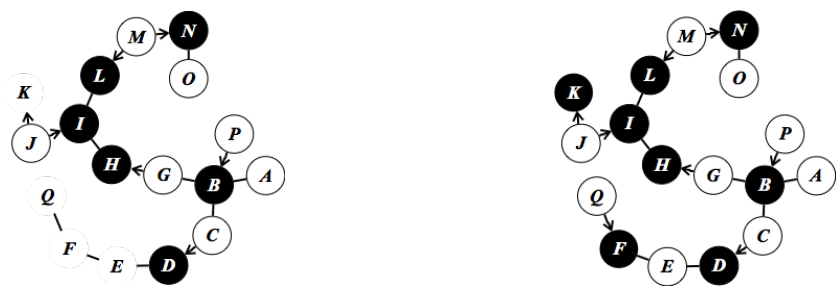

Fig. 4. A contraction-based entailment resolution (left) and a contraction-based semantic equivalence resolution (right).

Both resolutions change their attack relations by changing some mutual attacks to one-directional. Note that the entailment resolution has the contraction $\Sigma \backslash\{\neg f\}$, but the semantic equivalence resolution has the expansion $\Sigma \cup\{e, \neg k, q\}$. Note that a semantic equivalence resolution is stricter than an entailment resolution, and an entailment resolution is stricter than a satisfiability resolution. Each type of resolution corresponds to agent's attitude of how much closeness between cognitive and semantic acceptability they require.

\footnotetext{
${ }^{7}$ The rightmost graph is not an unsatisfiable core when complete extensions are assumed.
} 


\section{Conclusions and Discussion}

Our motivation of this work is that, as with us human beings, agent's perceptive development can be achieved by resolving the gaps between cognitive and semantic acceptability. In this paper, we introduced equilibrium-based satisfiability resolutions, entailment resolutions and semantic equivalence resolutions. We showed generality of our proposal by proving existence conditions of their resolutions. We demonstrated its applicability to online forums. The limitations of this paper is that our equilibriumbased resolutions mention nothing about sentences additional arguments should have. Our expansion-based resolutions only mention about an attack relation that additional arguments should have with other arguments.

This paper relates to research studies of belief revisions on abstract argumentation frameworks (AFs). [11] proposes a typology of revisions of AFs in terms of changes on extensions. [6] addresses a problem of how to modify AFs so that a desired set of arguments becomes an extension of revised AFs. [7] handles changes of AFs by introducing several revision operators that satisfy AGM (Alchourrón, Gärdenfors, and Makinson) postulates [2]. These research studies commonly focus on changes of AFs. In contrast, [16] focuses on belief revisions on agents' perceptions about acceptability status of arguments, and provide web-platform ArgTech that alerts users to irrational agents' perceptions through incompatibility checking with the complete labelling [3]. $[9,15]$ focus on changes of both AFs and agents' perceptions, and deal with resolutions of incoherence of agent's beliefs by expanding AF and constraints on its outcomes. In contrast, we primarily focus on balancing between perceptions (or constraints) and outcomes of argumentation. We thus introduce equilibrium mechanisms to handle simultaneous changes of them although the related work handles, at most, each of them. Notice that the resolutions analysed in Section 4.2 can only be achieved as an equilibrium, and a change of either AF or agents' perceptions is a special case of our equilibrium-based resolutions. Moreover, although the related work focuses on expansions and incoherence ("satisfiability" in this paper), this paper further deals with contraction and introduces entailment and semantic equivalence resolutions.

\section{A Proofs}

Proof. (Theorem 1) $\left(\Rightarrow\right.$ ) Assume $\Sigma \models \neg \Phi_{A F} . \Phi_{A F_{\uparrow}} \models \Phi_{A F}$ holds because $\operatorname{att}\left(A F_{\uparrow}\right) \supseteq$ $\operatorname{att}(A F)$ holds, for all $A F_{\uparrow} \sqsupseteq A F$. Thus, $\Sigma \models \neg \Phi_{A F_{\uparrow}}$ holds. Since $\varepsilon\left(A F_{\uparrow}\right) \models \Phi_{A F_{\uparrow}}$ holds, $\left\{\Phi_{A F_{\uparrow}}\right\} \cup \Sigma$ is unsatisfiable and $\varepsilon\left(A F_{\uparrow}\right) \not \models \Sigma$ holds. Thus, there is neither minimally satisfiable framework nor minimally entailing framework with respect to $\Sigma$. $(\Leftarrow)$ It is sufficient to show entailment resolutions. Assuming that $\Sigma \not \forall \neg \Phi_{A F}$ holds, there is a model $M$ of $\Sigma$ and $\Phi_{A F}$, i.e., $M \models \Sigma \cup\left\{\Phi_{A F}\right\}$. We want to show that there is $A F_{\uparrow} \sqsupseteq A F$ such that, for all $x \in M$ (resp. $x \notin M), \varepsilon\left(A F_{\uparrow}\right) \models x$ (resp. $\varepsilon\left(A F_{\uparrow}\right) \models \neg x$ ) hold. Consider $A F_{\Uparrow}$ adding a new argument $Y$ attacking only all arguments $X$ where $x \notin M$ holds. Obviously, $\varepsilon\left(A F_{\Uparrow}\right) \models \neg x$, for all $x \notin M$. Consider $A F_{\uparrow}$ adding another new argument $Z$ attacking only all arguments attacking $X$ where $x \in M$ holds. Here, since $M \mid=\Phi_{A F}$ holds, for all $(X, Y) \in \operatorname{att}(A F)$, if $y \in M$ then $x \notin M$, and vice versa. Thus, $\varepsilon\left(A F_{\uparrow}\right) \mid=x$, for all $x \in M$.

Proof. (Theorems 2, 3, 4, 5 and 6 (Sketch)) Similar to Theorem 1 or obvious from the case $A F=\langle\emptyset, \emptyset\rangle$ or $\Sigma=\emptyset$. 


\section{Acknowledgments}

The authors thank Dr. Martin Caminada for his valuable discussion. This work was supported by JSPS KAKENHI Grant Number 15KT0041.

\section{References}

[1] CreateDebate: Debate forum, online debate community. www.createdebate.com/, 2006.

[2] C. E. Alchourrón, P. Gärdenfors, and D. C. Makinson. On the logic of theory change: Partial meet contraction and revision functions. Journal of Symbolic Logic, 50(2):510-530, 1985.

[3] P. Baroni, M. Caminada, and M. Giacomin. An introduction to argumentation semantics. The Knowledge Engineering Review, 26:365-410, 2011.

[4] P. Baroni and M. Giacomin. On principle-based evaluation of extension-based argumentation semantics. Artificial Intelligence, 171(10-15):675-700, 2007.

[5] P. Baroni, M. Giacomin, and G. Guida. Scc-recursiveness: a general schema for argumentation semantics. Artificial Intelligence, 168(1-2):162-210, 2005.

[6] R. Baumann and G. Brewka. Expanding argumentation frameworks: Enforcing and monotonicity results. In Proc. of the 3rd International Conference on Computational Models of Argument, pages 75-86, 2010.

[7] R. Baumann and G. Brewka. AGM meets abstract argumentation: Expansion and revision for Dung frameworks. In Proc. of the 24th International Conference on Artificial Intelligence, pages 2734-2740, 2015.

[8] P. Besnard and S. Doutre. Checking the acceptability of a set of arguments. In Proc. of the 10th International Workshop on Non-Monotonic Reasoning, pages 59-64, 2004.

[9] R. Booth, S. Kaci, T. Rienstra, and L. V. D. Torre. A logical theory about dynamics in abstract argumentation. In Proc. of the 7th International Conference on Scalable Uncertainty Management, pages 148-161, 2013.

[10] M. Caminada. Semi-stable semantics. In Proc. of the 1st International Conference on Computational Models of Argument, pages 121-130, 2006.

[11] C. Cayrol, F. D. de Saint-Cyr, and M. C. Lagasquie-Schiex. Change in abstract argumentation frameworks: Adding an argument. Journal of Artificial Intelligence Research, 38:49-84, 2010.

[12] S. Coste-Marquis, C. Devred, and P. Marquis. Prudent semantics for argumentation frameworks. In Proc. of the 17th International Conference on Tools with Artificial Intelligence, pages 568-572, 2005.

[13] P. M. Dung. On the acceptability of arguments and its fundamental role in nonmonotonic reasoning, logic programming, and $n$-person games. Artificial Intelligence, 77:321-357, 1995.

[14] P. M. Dung, P. Mancarella, and F. Toni. A dialectic procedure for sceptical, assumptionbased argumentation. In Proc. of the 1st International Conference on Computational Models of Argument, pages 145-156, 2006.

[15] T. Rienstra. Argumentation in Flux: Modelling Change in the Theory of Argumentation. Dissertations and theses : Doctoral thesis, University of Luxembourg, Luxembourg, Luxembourg, 2014.

[16] C. Schulz and D. Dumitrache. The argteach web-platform. In Proc. of the 6th International Conference on Computational Models of Argument, pages 475-476, 2016.

[17] F. H. van Eemeren, R. Grootendorst, and F. S. Henkemans. Fundamentals of Argumentation Theory: A Handbook of Historical Backgrounds and Contemporary Developments. Routledge, 1996.

[18] B. Verheij. Two approaches to dialectical argumentation: admissible sets and argumentation stages. In Proc. of the 8th Dutch Conference on Artificial Intelligence, pages 357-368, 1996. 